\title{
Maximum Upward Planar Subgraphs of Embedded Planar Digraphs ${ }^{\star}$
}

\author{
Carla Binucci, Walter Didimo, and Francesco Giordano \\ DIEI - Università degli Studi di Perugia \\ \{binucci, didimo, giordano\}@diei.unipg.it
}

\begin{abstract}
This paper presents an extensive study on the problem of computing maximum upward planar subgraphs of embedded planar digraphs: Complexity results, algorithms, and experiments are presented. Namely: (i) We prove that the addressed problem is NP-Hard; (ii) A fast heuristic and an exponential-time exact algorithm are described; (iii) A wide experimental analysis is performed to show the effectiveness of our techniques.
\end{abstract}

\section{Introduction}

The upward drawing convention is commonly used to display hierarchical structures so that all edges flow in a common direction according to their orientation. More precisely, let $G$ be a directed graph (also called a digraph); an upward drawing of $G$ is such that each edge is drawn as a simple Jordan curve monotonically increasing in the upward direction. In particular, a wide research effort has been devoted so far to the design of algorithms for computing upward planar drawings, i.e, upward drawings without crossings. Indeed, there is a general consensus that the number of crossings between edges is one of the most critical aesthetic requirements for the readability of a drawing. A digraph that admits an upward planar drawing is called an upward planar digraph. Fig. 1(a) and 1(b) show a planar digraph $G$ and an upward planar drawing of $G$. The planar digraph in Fig. 1(c) is not upward planar.

Bertolazzi et al. [2] proved that if a digraph $G$ with $n$ vertices has a fixed planar embedding, then testing whether $G$ admits an upward planar drawing that preserves its embedding can be done in $O\left(n^{2}\right)$ time. On the other side, Garg and Tamassia [10] proved that the upward planarity testing problem in the variable embedding setting (i.e., over all planar embeddings of the input digraph) is NP-Complete. In this scenario, several polynomial-time upward planarity testing algorithms have been described in the literature for specific sub-families of planar digraphs [381214], and exponential-time algorithms for the same problem can be found in [1411].

^ Research partially supported by the MIUR Project "MAINSTREAM". 


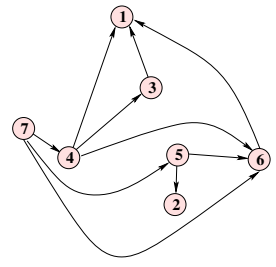

(a)

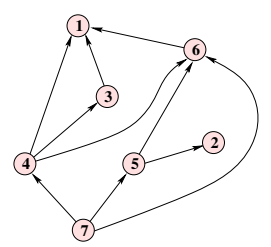

(b)

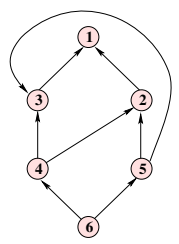

(c)

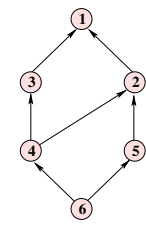

(d)

Fig. 1. (a) A planar digraph $G$ with a given planar (bimodal) embedding. (b) An embedding preserving upward planar drawing of $G$. (c) A digraph $G^{\prime}$ that is not upward planar; (d) A maximum upward planar subgraph of $G^{\prime}$.

When a planar digraph $G$ is not upward planar, an interesting problem that naturally arises is the one of computing a maximum upward planar subgraph of $G$, i.e., an upward planar subgraph with maximum number of edges. From the application side, solving this problem is important to find large hierarchical sub-structures in the digraph and to convey them visually. In the variable embedding setting, computing a maximum upward planar subgraph is NP-Hard as an immediate consequence of the hardness of the upward planarity testing problem [10]. If the embedding of the digraph is fixed, however, the complexity of the problem is still unknown. Recall that in this case the upward planarity testing problem is polynomially solvable 2 .

We present an extensive study on the problem of computing a maximum upward planar subgraph of an embedded planar digraph. Namely:

(i) We prove that finding a maximum upward planar subgraph of a planar digraph remains NP-Hard, even in the fixed embedding scenario (Section 3). Our proof uses a reduction from Planar 3-SAT [13. With the same reduction we also prove that finding the maximum bimodal subgraph of an embedded planar digraph is NP-Hard. Recall that an embedded digraph is bimodal if the incoming and the outgoing edges of each vertex never alternate (see, e.g., Fig. 1(a)]. Notice that the bimodality is necessary (but not sufficient) for the upward planarity.

(ii) Motivated by the above negative results, we describe both a polynomialtime heuristic and a branch-and-bound exact algorithm to compute a maximum upward planar subgraph of an embedded planar digraph (Section 44). The input digraph is not necessarily bimodal and acyclic. Our heuristic adopts a greedy approach for computing a large bimodal subgraph and then extracts from it an upward planar subgraph by using a combination of the techniques given in [1|2]. Notice that, in the variable embedding setting any heuristic that uses an upward planarity testing as a key tool would still require exponential time.

(iii) We perform a wide experimental study, which shows how our heuristic is pretty fast and effective in practice; it achieves the optimum in many cases and definitively outperforms a simple technique that incrementally tries to insert an edge per time while preserving upward planarity (Section 5). 


\section{Basic Definitions}

We assume familiarity with basic concepts of graph planarity and graph drawing [5. We denote by $G_{\Phi}$ an embedded planar digraph, i.e., a planar digraph $G$ with a given planar embedding $\Phi$, where $\Phi$ describes the set of (internal and external) faces for $G$ in the plane. For each vertex $v$ of $G, \Phi$ also fixes the circular clockwise ordering of the edges incident to $v$. An embedding preserving subgraph $G_{\Phi^{\prime}}^{\prime}$ of $G_{\Phi}$ is an embedded planar digraph obtained from $G_{\Phi}$ by removing a subset of its edges. Notice that, for each vertex $v$ of $G_{\Phi^{\prime}}^{\prime}$, the circular clockwise ordering of the edges incident to $v$ in $G_{\Phi^{\prime}}^{\prime}$ is the same as in $G_{\Phi}$.

A vertex $v$ of $G_{\Phi}$ is bimodal if all incoming edges of $v$ (and hence all outgoing edges of $v$ ) appear consecutive in the circular clockwise ordering around $v$. If all vertices of $G_{\Phi}$ are bimodal, $\Phi$ is called a planar bimodal embedding and $G_{\Phi}$ is called a planar bimodal embedded digraph. A planar digraph $G$ is bimodal if it admits a planar bimodal embedding. The digraph in Fig. 1(a) is a planar bimodal embedded digraph.

An upward planar drawing of $G_{\Phi}$ is a planar drawing of $G$ that preserves the embedding $\Phi$ and such that all the edges of $G$ are drawn as curves monotonically increasing in the upward direction. We say that $G_{\Phi}$ is upward planar if it admits an upward planar drawing. It is known that acyclicity and bimodality are necessary (but not sufficient) conditions for the upward planarity [2]. For example, the planar digraph in Fig. 1(c) is acyclic and bimodal, but it does not admit an upward planar drawing.

A maximum upward planar subgraph $G_{\Phi^{\prime}}^{\prime}$ of $G_{\Phi}$ is an embedding preserving subgraph of $G_{\Phi}$ with the following two properties: (a) $G_{\Phi^{\prime}}^{\prime}$ is upward planar; (b) $G_{\Phi^{\prime}}^{\prime}$ has the maximum number of edges among the embedding preserving subgraphs of $G_{\Phi}$ that are upward planar. Fig. 1(d) shows a maximum upward planar subgraph of the embedded digraph in Fig. 1(c)

\section{Complexity Results}

We define the Fixed Embedding Maximum Upward Planar Subgraph (FE-MUPS) problem as follows.

Problem FE-MUPS: Given a pair $\left\langle G_{\Phi}, K\right\rangle$, where $G_{\Phi}=(V, E)$ is an embedded planar digraph and $K$ is an integer number such that $0<K<|E|$, does $G_{\Phi}$ admit an embedding preserving subgraph $G_{\Phi^{\prime}}^{\prime}=\left(V, E^{\prime}\right)$ such that $\left|E^{\prime}\right|=K$ and $G_{\Phi^{\prime}}^{\prime}$ is upward planar?

We prove that FE-MUPS is NP-Complete. The hardness proof uses a reduction from Planar 3-SAT, a restricted version of 3-SAT 9. To fix notation, we recall the definitions of 3 -SAT and Planar 3 -SAT.

Problem 3-SAT: Let $\langle X, C, \Psi\rangle$ be a tuple such that $X=\left\{x_{1}, \ldots, x_{n}\right\}$ is a set of boolean variables, $C=\left\{c_{1}, \ldots, c_{m}\right\}$ is a set of clauses such that $c_{i}=$ $\left(\ell_{i, 1} \vee \ell_{i, 2} \vee \ell_{i, 3}\right)(i \in\{1, \ldots, m\})$, each $\ell_{i, j}(j \in\{1,2,3\})$ is a literal that can be equal either to a boolean variable $x \in X$ or to the negation $\bar{x}$ of a boolean 
variable, and $\Psi$ is a boolean formula of the form $\Psi=c_{1} \wedge c_{2} \wedge \cdots \wedge c_{m}$. Is there a truth assignment for the variables of $X$ such that $\Psi$ is satisfied?

An instance of Planar 3-SAT is any instance of 3-SAT for which a special graph $H_{\Psi}$, associated with $\Psi$, is planar. The question of Planar 3-SAT is the same as for 3-SAT. Graph $H_{\Psi}$ is defined as follows (refer to Fig. 2): For each variable $x \in X, H_{\Psi}$ has a vertex associated with $x$ and a vertex associated with its negation $\bar{x} H_{\Psi}$ has a vertex for each clause $c \in C$, called a clausevertex. $H_{\Psi}$ has an edge $\left(\ell_{i, j}, c_{i}\right)$ for each literal $\ell_{i, j}$ of $c_{i}(i \in\{1, \ldots, m\}, j \in$ $\{1,2,3\}) . H_{\Psi}$ has an edge $(x, \bar{x})$ for each variable $x \in X . H_{\Psi}$ has a cycle of edges $\left(x_{1}, x_{2}\right),\left(x_{2}, x_{3}\right), \ldots,\left(x_{n-1}, x_{n}\right),\left(x_{n}, x_{1}\right)$.

$$
\begin{aligned}
& \Psi=c_{1} \wedge c_{2} \wedge c_{3} \wedge c_{4} \wedge c_{5} \\
& c_{1}=\left(x_{1} \vee x_{2} \vee \bar{x}_{3}\right) \\
& c_{2}=\left(x_{1} \vee \bar{x}_{3} \vee x_{5}\right) \\
& c_{3}=\left(\bar{x}_{1} \vee \bar{x}_{2} \vee \bar{x}_{5}\right) \\
& c_{4}=\left(\bar{x}_{2} \vee x_{3} \vee \bar{x}_{4}\right) \\
& c_{5}=\left(x_{3} \vee x_{4} \vee x_{5}\right)
\end{aligned}
$$

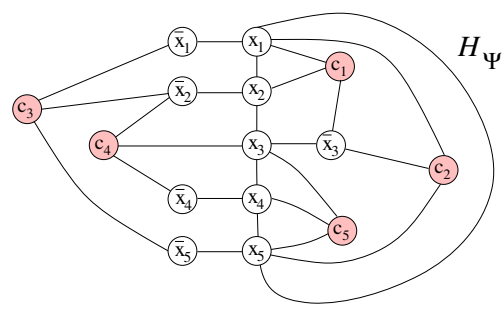

(b)

Fig. 2. (a) An instance of Planar 3-SAT. (b) The planar graph $H_{\Psi}$ associated with $\Psi$.

\section{Lemma 1. Problem FE-MUPS is NP-Hard.}

Proof. We define a polynomial-time reduction from a generic instance $\langle X, C, \Psi\rangle$ of Planar 3-SAT to an instance $\left\langle G_{\Phi}, K\right\rangle$ of FE-MUPS, and then we show that $\Psi$ is satisfied if and only if $G_{\Phi}$ has an embedding preserving upward planar subgraph with $K$ edges. Let $H_{\Psi}$ be the planar graph associated with $\Psi$ along with an arbitrary planar embedding. The embedded planar digraph $G_{\Phi}$ is constructed from the embedded graph $H_{\Psi}$ as follows: (refer to Fig. 3 for an illustration):

(a) Remove the cycle of edges $\left(x_{1}, x_{2}\right),\left(x_{2}, x_{3}\right), \ldots,\left(x_{n-1}, x_{n}\right),\left(x_{n}, x_{1}\right)$; assign to each edge $\left(\ell_{i, j}, c_{i}\right)$ a label equal to $\ell_{i, j}(i \in\{1, \ldots, m\}, j \in\{1,2,3\})$, and then contract each edge $\left(x_{r}, \bar{x}_{r}\right),(r \in\{1, \ldots, n\})$. Denote by $y_{r}$ the vertex that originates from the contraction of $\left(x_{r}, \bar{x}_{r}\right)$, and orient every edge $\left(y_{r}, c_{i}\right)$ from $y_{r}$ to $c_{i}$ (see Fig. $3(\mathrm{a})$ ). The edges $\left(y_{r}, c_{i}\right)$ will be called variable edges.

(b) For each clause vertex $c_{i}$ add six new vertices $c_{i, 1}, \ldots, c_{i, 6}$ and the six directed edges $\left(c_{i}, c_{i, h}\right)(i \in\{1, \ldots, m\}, h \in\{1, \ldots, 6\})$. The new edges are embedded around $c_{i}$ in such a way that there are exactly two of them between every pair of circularly consecutive incoming edges of $c_{i}$. Also, for each vertex $y_{r}(r \in\{1, \ldots, n\})$ add two new vertices $y_{r, 1}, y_{r, 2}$ and the two directed edges

\footnotetext{
${ }^{1}$ In the original formulation of the Planar 3-SAT problem there is only one vertex per variable, which represents both $x$ and $\bar{x}$. Lichtenstein 13 proved that the Planar 3 -SAT problem remains NP-Complete if one considers distinct vertices for $x$ and $\bar{x}$ (see Lemma 1 of [13); we use this variant.
} 
$\left(y_{r, 1}, y_{r}\right),\left(y_{r, 2}, y_{r}\right)$; these two edges are embedded around $y_{r}$ in such a way that they separate the (possibly empty) sequence of edges labeled $x_{r}$ from the (possibly empty) sequence of edges labeled $\bar{x}_{r}$ (see Fig. $3(\mathrm{~b})$. Every edge added during this step will be called a dangling edge.

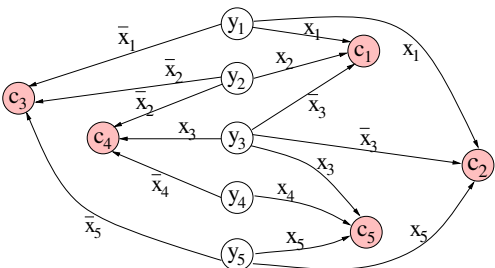

(a)

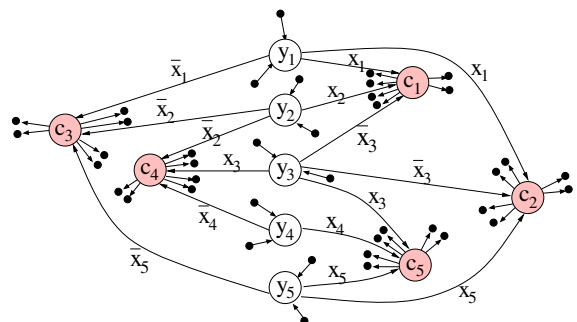

(b)

Fig. 3. Reduction from the graph associated with an instance of Planar 3-SAT to the graph of an instance of FE-MUPS. The reduction is done from planar graph $H_{\Psi}$ of Fig. 2(b) The edges incident to the small black vertices are the dangling edges.

The transformation described above to construct $G_{\Phi}$ from $H_{\Psi}$ preserves the planarity, and thus $G_{\Phi}$ is an embedded planar digraph. Also, this transformation can be easily performed in $O(m+n)$ time, i.e., in a time linear in the size of $\Psi$. To complete the reduction, we have to fix a value for $K$. We choose $K=7 m+2 n$.

Before proving that $\Psi$ is satisfiable if and only if $G_{\Phi}$ has an embedding preserving upward planar subgraph with $K$ edges, we prove that, with our choice of $K$, every embedding preserving subgraph $G_{\Phi^{\prime}}^{\prime}$ of $G_{\Phi}$ with $K$ edges is upward planar if and only if $G_{\Phi^{\prime}}^{\prime}$ is bimodal. Clearly, if $G_{\Phi^{\prime}}^{\prime}$ is upward planar then it is necessarily bimodal. Suppose vice-versa that $G_{\Phi^{\prime}}^{\prime}$ has $K$ edges and is bimodal. We claim that $G_{\Phi^{\prime}}^{\prime}$ contains necessarily $m$ variable edges and all the $6 m+2 n$ dangling edges. Namely, $G_{\Phi}$ consists of $3 m$ variable edges (3 edges incident to each clause-vertex) and $6 m+2 n$ dangling edges: If $G_{\Phi^{\prime}}^{\prime}$ had less than $m$ variable edges, then it would have less than $K$ edges in total. On the other hand, suppose that $G_{\Phi^{\prime}}^{\prime}$ consists of $m+h$ variable edges $(1 \leq h \leq 2 m)$. Each variable edge is an incoming edge of a clause-vertex; also, if a clause-vertex has $1+p$ incoming edges in $G_{\Phi^{\prime}}^{\prime}(0 \leq p \leq 2)$, then it has at most $6-2 p$ outgoing edges (i.e., incident dangling edges) in $G_{\Phi^{\prime}}^{\prime}$, otherwise the bimodality of the clause-vertex would be violated. Since there are exactly $m$ clause-vertices in $G_{\Phi}$, it follows that if $G_{\Phi^{\prime}}^{\prime}$ consisted of $m+h$ variable edges, the number of dangling edges that we can hope to have in $G_{\Phi^{\prime}}^{\prime}$ without violating the bimodality would be at most $6 m-2 h+2 n$, and therefore the number of edges of $G_{\Phi^{\prime}}^{\prime}$ would be at most $m+h+6 m-2 h+2 n=7 m+2 n-h<K$, which contradicts the hypothesis that $G_{\Phi^{\prime}}^{\prime}$ has $K$ edges. Hence, if $G_{\Phi^{\prime}}^{\prime}$ is an embedding preserving bimodal subgraph of $G_{\Phi}$ with $K$ edges, it consists of exactly $m$ variable edges and of all $6 m+2 n$ dangling edges, which proves the claim. ¿From the bimodality of $G_{\Phi^{\prime}}^{\prime}$, there is exactly one variable edge incident to each clause-vertex and each vertex 
$y_{r}(r \in\{1, \ldots, n\})$ cannot have incident edges labeled $x_{r}$ and incident edges labeled $\bar{x}_{r}$ at the same time. Observe now that the undirected underlying graph of such a bimodal subgraph $G_{\Phi^{\prime}}^{\prime}$ does not contain simple cycles; indeed, a simple cycle can only consist of variable edges, but each variable edge connects some clause-vertex $c_{i}$ to some vertex $y_{r}$, and each clause-vertex has only one incident variable edge in $G_{\Phi^{\prime}}^{\prime}$. Since every planar embedded bimodal digraph whose underlying graph is acyclic is also upward planar, we conclude that $G_{\Phi^{\prime}}^{\prime}$ is upward planar.

We now prove that if $\Psi$ is satisfiable then there exists an embedding preserving upward planar subgraph of $G_{\Phi}$ with $K$ edges. For what we have proved so far, it is sufficient to construct, from the truth assignment of $\Psi$, an embedding preserving bimodal subgraph $G_{\Phi^{\prime}}^{\prime}$ with $K$ edges. $G_{\Phi^{\prime}}^{\prime}$ consists of all vertices and all dangling edges of $G_{\Phi}$; furthermore, add to $G_{\Phi^{\prime}}^{\prime} m$ variable edges as follows: For each clause $c_{i}$ of $\Psi(i \in\{1, \ldots, m\})$, select exactly one literal $\ell_{i, j}$ of $c_{i}$ having value true $(j \in\{1,2,3\})$ and add to $G_{\Phi^{\prime}}^{\prime}$ the variable edge with label $\ell_{i, j}$, incident to clause-vertex $c_{i}$. By construction, $G_{\Phi^{\prime}}^{\prime}$ has $m+6 m+2 n=7 m+2 n=K$ edges, and the bimodality of $G_{\Phi^{\prime}}^{\prime}$ is implied by two properties: (i) each clause-vertex has exactly one incoming edge; (ii) each vertex $y_{r}(r \in\{1, \ldots, n\})$ has at most one circular sequence of consecutive outgoing edges and two circularly consecutive incoming edges, because $x_{r}$ and $\bar{x}_{r}$ cannot be true at the same time.

Suppose vice-versa that $G_{\Phi^{\prime}}^{\prime}$ is an embedding preserving upward planar subgraph with $K$ edges. As proved above, $G_{\Phi^{\prime}}^{\prime}$ has exactly one variable edge incident to each clause-vertex and it contains all dangling edges of $G_{\Phi}$. This implies that each vertex $y_{r}(r \in\{1, \ldots, n\})$ cannot have incident edges labeled $x_{r}$ and incident edges labeled $\bar{x}_{r}$ at the same time. Therefore, a valid truth assignment that satisfies $\Psi$ can be derived by simply assigning value true to those literals that correspond to labels of the variable edges of $G_{\Phi^{\prime}}^{\prime}$.

The proof of the next lemma is easy and it is omitted for space limitations.

Lemma 2. Problem FE-MUPS belongs to NP.

From Lemma 1 and Lemma 2 we have the following result.

\section{Theorem 1. Problem FE-MUPS is NP-Complete.}

With the reduction used in the proof of Lemma 1 finding an embedding preserving upward planar subgraph of $G_{\Phi}$ with $K$ edges is equivalent to find an embedding preserving bimodal subgraph of $G_{\Phi}$ with $K$ edges. This immediately implies that also finding a maximum embedding preserving bimodal subgraph of an embedded digraph is a hard problem. More formally, the problem Fixed Embedding Maximum Bimodal Planar Subgraph is defined as follows.

Problem FE-MBPS: Given a pair $\left\langle G_{\Phi}, K\right\rangle$, where $G_{\Phi}=(V, E)$ is an embedded planar digraph and $K$ is an integer number such that $0<K<|E|$, does $G_{\Phi}$ admit an embedding preserving subgraph $G_{\Phi^{\prime}}^{\prime}=\left(V, E^{\prime}\right)$ such that $\left|E^{\prime}\right|=K$ and $G_{\Phi^{\prime}}^{\prime}$ is bimodal? 
The NP-Hardness of FE-MBPS is implied by the proof of Lemma 11 Also, it is easy to see that FE-MBPS belongs to NP.

Theorem 2. Problem FE-MBPS is NP-Complete.

\section{Algorithms}

Motivated by Theorem 1, we designed a polynomial-time heuristic (Subsection 4.1) and an exponential-time exact algorithm (Subsection 4.2) for computing maximum upward planar subgraphs of embedded planar digraphs. Both these algorithms accept in input an embedded planar digraph $G_{\Phi}$ that is not necessarily acyclic and bimodal. Before describing our techniques, we observe that a straightforward algorithm to compute a maximal upward planar subgraph of $G_{\Phi}$ is as follows: Remove all edges from $G_{\Phi}$ and then try to reinsert an edge per time; each time a new edge $e$ is selected for possible insertion, an upward planarity testing algorithm for fixed embedding is applied on the current subgraph plus edge $e$; if the test is positive, $e$ is added to the subgraph otherwise $e$ is definitively discarded. Such an algorithm, which we refer to as SimpleAlgorithm, is easy to implement and runs in time $O\left(n^{3}\right)$ if one uses the $O\left(n^{2}\right)$-time upward planarity testing technique of Bertolazzi et al. [2]. However, SimpleAlgorithm is rather slow in practice, because it applies the upward planarity testing algorithm for each edge of $G_{\Phi}$ (see also Section 5). Instead, we designed an algorithm that is much faster in practice and also more effective than SimpleAlgorithm. Furthermore, it represents a key basic tool for the design of the exact algorithm.

\subsection{A Fast and Effective Heuristic}

Our heuristic, which we call BendAlgorithm, computes a maximal upward planar subgraph of the input digraph $G_{\Phi}=(V, E)$ in three main steps, described below.

- Step 1 computes an embedding preserving subgraph $G_{\Phi^{\prime}}^{\prime} \subseteq G_{\Phi}$ that is bimodal and that contains as much edges as possible. Since by Theorem 2 the problem of finding a maximum bimodal subgraph of $G_{\Phi}$ is NP-Hard, we designed for this step an algorithm that just computes a maximal bimodal subgraph $G_{\Phi^{\prime}}^{\prime}$. This algorithm first removes a minimal subset of edges from $G_{\Phi}$ until the digraph becomes bimodal, and then it tries to reinsert each of the removed edges in a random order; an edge is reinserted iff it does not violate the bimodality. The removal of a minimal subset of edges to get the bimodality is based on a greedy procedure. Namely, let $v$ be a vertex of $G_{\Phi}$ and let $E(v)$ be the circular sequence of edges incident to $v$. If $v$ is not bimodal, denote by $E^{\prime}(v)$ a minimum subset of edges of $E(v)$ whose removal makes $v$ bimodal. We associate with $v$ a cost $c(v)=\left|E^{\prime}(v)\right|$. Subset $E^{\prime}(v)$ is computed in time $O\left(|E(v)|^{2}\right)$ by considering all possible splits of $E(v)$ into two linear lists, $E_{1}(v)$ and $E_{2}(v)$, and by adding to $E^{\prime}(v)$ all the incoming edges of $v$ that belong to $E_{1}(v)$ and all the outgoing edges of $E(v)$ that belong to $E_{2}(v)$. The removal of the edges of $E^{\prime}(v)$ from $G_{\Phi}$ makes $v$ bimodal, because the remaining edges of $E_{1}(v)$ will be outgoing 
edges of $v$, while the remaining edges of $E_{2}(v)$ will be incoming edges of $v$. Since $\sum_{v \in V}|E(v)|^{2} \leq\left(\sum_{v \in V}|E(v)|\right)^{2}=(2|E|)^{2}$, all costs $c(v)$ can be computed in time $O\left(|E|^{2}\right)$. At each phase of the greedy procedure, a non-bimodal vertex $v$ with minimum $\operatorname{cost} c(v)$ is selected, and all the edges of $E^{\prime}(v)$ are temporarily removed. Also, for each edge $(u, v)$ or $(v, u)$ in $E^{\prime}(v)$, cost $c(u)$ and set $E^{\prime}(u)$ are updated. We use a binary heap priority queue to efficiently store and update the costs of the non bimodal vertices and to extract their minimum value at each phase. Hence, the greedy procedure consists of at most $|V|$ phases, each requiring $O\left(|E|^{2}\right)=O\left(|V|^{2}\right)$ time. The edges temporarily removed during the greedy procedure are possibly reinserted incrementally, one per time; this is done in $O\left(|E|^{2}\right)$, because the bimodality can be tested efficiently for each edge reinsertion. Hence, Step 1 takes $O\left(|V|^{3}\right)$ time.

- Step 2 temporarily removes from $G_{\Phi^{\prime}}^{\prime}$ a minimal number of edges in order to get an upward planar subgraph $G_{\Phi^{\prime \prime}}^{\prime \prime} \subseteq G_{\Phi^{\prime}}^{\prime}$. To do this, it applies a global strategy. More precisely, in [1] the concept of quasi-upward planar drawing of an embedded bimodal digraph is introduced. Roughly speaking, a quasi-upward planar drawing is an upward drawing that allows some bends along the edges, where a bend represents an inversion of direction of an edge. If one removes all the bent edges in a quasi-upward planar drawing, the remaining drawing is upward planar. In [1] an $O\left(|V|^{2} \log |V|\right)$ flow-based algorithm is described for the computation of a quasi-upward planar drawing of an embedded bimodal digraph, having the minimum number of bends. In general this is not equal to determine the minimum number of edges whose removal leads to the upward planarity, but it typically behaves as an effective heuristic to this aim. Thus, to compute $G_{\Phi^{\prime \prime}}^{\prime \prime}$ we apply on $G_{\Phi^{\prime}}^{\prime}$ the flow-based algorithm of [1] and temporarily remove the bent edges.

- Step 3 tries to incrementally reinsert in a random order the edges removed in Step 2, by testing each edge reinsertion for upward planarity. Each test is done by applying the $O\left(|V|^{2}\right)$-time algorithm of Bertolazzi et al. 2. The number of tests executed is equal to the number of edges removed during Step 2, which will be proved to be rather small in practice (see Section 5 ).

The following lemma summarizes the discussion above.

Lemma 3. Let $G_{\Phi}$ be an embedded planar digraph and let $n$ be the number of vertices of $G_{\Phi}$. BendAlgorithm computes a maximal upward planar subgraph of $G_{\Phi}$ in $O\left(n^{3}\right)$ time.

\subsection{An Exact Algorithm}

Our exact algorithm, which we call BBAlgorithm, is based on a branch-andbound technique. Let $G_{\Phi}=(V, E)$ be the input digraph and let $E=\left\{e_{1}, \ldots, e_{m}\right\}$ be the set of its edges. To encode any subset $E^{\prime}$ of $E$ we use an array of binary variables $X_{E^{\prime}}=\left\{x_{1}, x_{2}, \ldots, x_{m}\right\}$, where $x_{i}=0$ if edge $e_{i}$ does not belong to $E^{\prime}$, and $x_{i}=1$ if $e_{i}$ belongs to $E^{\prime}$. The optimal solution is an array $X_{E^{\prime}}$ such that subgraph $\left(V, E^{\prime}\right)$ is upward planar and the number of variables of $X_{E^{\prime}}$ having value 0 is minimized (in the following a variable of value 0 will be called a zero variable). 
The branch-and-bound tree $T$ is a complete binary tree with levels $0,1, \ldots, m$, where the leaves represent all subsets of $E$. Each leaf of $T$ is an array $X_{E^{\prime}}$, for some $E^{\prime} \subseteq E$. An internal node $\mu$ of $T$ at level $i(1 \leq i<m)$ is associated with an array of $i$ binary variables $X_{\mu}=\left\{x_{1}^{(\mu)}, x_{2}^{(\mu)}, \ldots, x_{i}^{(\mu)}\right\}$; in the subtree $T_{\mu}$ rooted at $\mu$, each leaf is an array $X_{E^{\prime}}=\left\{x_{1}^{(\mu)}, x_{2}^{(\mu)}, \ldots, x_{i}^{(\mu)}, x_{i+1}, \ldots, x_{m}\right\}$, i.e., it represents a subset $E^{\prime} \subseteq E$ such that $e_{j} \in E^{\prime}$ iff $x_{j}^{(\mu)}=1(1 \leq j \leq i)$.

The algorithm visits $T$ from the root to the leaves, and an array $\bar{X}$ corresponding to the current best solution is kept updated during the visit ( $\bar{X}$ always coincides with a leaf of $T$ ). At the beginning of the visit, $\bar{X}$ is set equal to a solution computed with the BendAlgorithm. Each time a new internal node $\mu$ at a level $i$ is visited, the subgraph induced by the non-zero variables $x_{1}^{(\mu)}, x_{2}^{(\mu)}, \ldots, x_{i}^{(\mu)}$ is tested for upward planarity; if the test is negative $T_{\mu}$ is cut and it will be not visited in the following; otherwise an upper bound $u(\mu)$ and a lower bound $l(\mu)$ to the number of zero variables contained in any leaf of $T_{\mu}$ are computed. In particular, $u(\mu)$ will correspond to the number of zero variables in some leaf $X_{E^{\prime}}$ of $T_{\mu}$, representing an upward planar subgraph. If $u(\mu)$ is smaller than the number of zero variables of $\bar{X}$, then $\bar{X}$ is updated with $X_{E^{\prime}}$. If $l(\mu)$ is greater than or equal to the number of zero variables of $\bar{X}$, then subtree $T_{\mu}$ is cut and it will be not visited. To visit $T$ the algorithm applies a depth-first-search, which uses a stack to store the visited nodes. At any time of the visit, the number of nodes stored in the stack is $O(m)$ (i.e., order of the depth of $T$ ). At the end of the visit, $\bar{X}$ will represent an optimal solution. We now describe how $u(\mu)$ and $l(\mu)$ are computed.

The upper bound $u(\mu)$ is determined by applying a technique similar to the one used by BendAlgorithm. Namely, we first complete the array $X_{\mu}$ to an array $X_{E^{\prime}}$ corresponding to a maximal bimodal subgraph of the input digraph; this is done by incrementally testing for insertion all the edges $e_{i+1}, \ldots, e_{m}$. After that, we apply on the subgraph associated with $X_{E^{\prime}}$ the flow-based algorithm in [1], so to get a quasi-upward planar drawing; the bent edges are temporarily removed and then incrementally tested for possible reinsertion with the algorithm in [2]. To guarantee that none of the edges $e_{j}$ for which $x_{j}^{(\mu)}=1$ is removed $(1 \leq j \leq i)$, we constrain these edges to have no bend in the computed quasi-upward planar drawing; this is done by imposing a suitable flow constraint.

The lower bound $l(\mu)$ is computed by performing $m-i$ steps. For each $h=$ $i+1, \ldots, m$, we test if the subgraph induced by the non-zero variables of $X_{\mu}$ plus edge $e_{h}$ is bimodal and upward planar; a negative test implies that $e_{h}$ is not contained in any of the upward planar subgraphs represented by the leaves of $T_{\mu}$, and then we increment $l(\mu)$ by one unit.

\section{Experimental Study}

We implemented SimpleAlgorithm, BendAlgorithm, and BBAlgorithm and experimentally compared their performances. For the implementation we used 
$\mathrm{C}++$ and the GDToolkit graph drawing library 2 . The experiments were executed under Linux OS, on a machine with an Intel Centrino 1.66 GHz and 2GB of RAM. For the experiments we used three different test suites of connected planar digraphs. The first test suite, which we call BimodalAcyclic, is a set of 800 embedded planar digraphs that are bimodal and acyclic, and having number of vertices in $\{10,20, \ldots, 200\}$. Since we observed that the performances of our algorithms are strongly influenced by the density of the input digraph, we generated 10 different digraphs for each fixed number of vertices and distinct density value in $\{1.2,1.6,2.0,2.4\}$. Each digraph in BimodalAcyclic was obtained by randomly generating an embedded upward planar digraph with the algorithm described in [7] and then changing at random the orientation of the $50 \%$ of the edges, while preserving bimodality and acyclicity; if the resulting digraph was still upward planar it was discarded and generated again. The second test suite, which we call Any, consists of 800 embedded planar digraphs with no additional restriction. As a consequence, a digraph in this test suite is in general not bimodal and not acyclic. Again, digraphs in Any have number of vertices in $\{10,20, \ldots, 200\}$ and density in $\{1.2,1.6,2.0,2.4\}$. Each digraph in Any was generated at random by first generating a tree and then adding a number of edges between the vertices of the tree, until the desired value of density was achieved. Each edge was then randomly oriented with a uniform probability distribution. Finally, we used a third test suite, called Rome, derived from the well known set of graphs defined in [6], and often recognized as "Rome Graphs". The Rome Graphs have number of vertices in $[10,100]$, are not directed and, in general, not planar. At the web site http://www.dia.uniroma3.it/ gdt/, an oriented version of the Rome Graphs is available, where each edge has been oriented at random. We randomly selected 50 of these digraphs for each fixed number of vertices in $\{10,15,20, \ldots, 95,100\}$, for a total of 1000 digraphs. Then, for each of these digraphs, we planarized it (by possibly adding dummy vertices) and randomly chose a planar embedding. The average density of such digraphs is about 1.4.

We first compared BendAlgorithm and SimpleAlgorithm on the first two test suites. BendAlgorithm runs pretty fast and outperforms SimpleAlgorithm. Indeed, SimpleAlgorithm executes the $O\left(n^{2}\right)$ upward planarity testing described in 2] for each edge insertion, while BendAlgorithm applies the same test only for those edges having some bends in the quasi-upward drawing computed in Step 2, which are a small percentage of the whole set of edges (around $2.5 \%$ for low density digraphs and about $8-10 \%$ for high density digraphs). Also, the running times of Steps 1 and 2 of BendAlgorithm are in practice negligible with respect to the time taken from the reinsertion process in Step 3, even for the digraphs in Any (Steps 1-2 take about 0.01 seconds for graphs of 200 vertices and density 1.6, and 0.2 seconds for graphs of 200 vertices and density 2.4).

The effectiveness of the two heuristics is measured in terms of the size of their solutions, i.e., the number of edges in the computed maximal upward planar subgraphs; we express such a size as a percentage of the whole set of edges of the input digraph. We observed that this percentage does not depend on

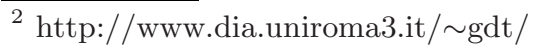




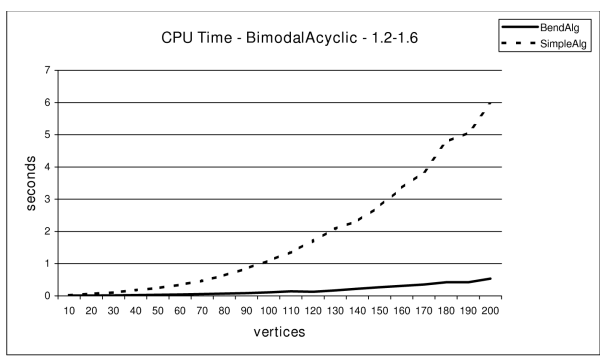

(a)

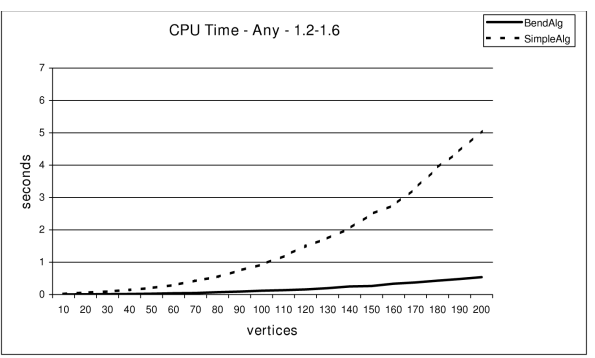

(b)

Fig. 4. Average CPU time of BendAlgorithm (solid line) and SimpleAlgorithm (dashed line) on the BimodalAcyclic and Any instances. For space reasons, we group the data on densities 1.2, 1.6 and omit the data for higher densities.

the number of vertices of the input digraph, but only on the density of the digraphs (see Table 1). One can observe that BendAlgorithm is more effective than SimpleAlgorithm and the difference in the effectiveness of the two heuristics grows with the increasing of the density. We also observe that the solutions computed for the digraphs in Any have smaller size than those computed for the digraphs in BimodalAcyclic. This because the digraphs in Any are typically not bimodal and not acyclic, and therefore require more edges to be deleted. The computations on the Rome test suite confirmed the behavior of the two heuristics on the digraphs with densities 1.2-1.6 in the Any test suite; BendAlgorithm and SimpleAlgorithm inserted $93.02 \%$ and $90.18 \%$ of the total edges, respectively.

Table 1. Average percentage (size) of number of edges in the solutions computed by BendAlgorithm and SimpleAlgorithm for each density value. The differences between the sizes of the solutions computed with the two algorithms are also shown.

\begin{tabular}{|c|c|c|c||c|c|c|c|}
\hline \multicolumn{3}{|c||}{ BimodalAcyclic } & \multicolumn{4}{c|}{ Any } \\
\hline \hline Density & BendAlg & SimpleAlg & Diff & Density & BendAlg & SimpleAlg & Diff \\
\hline 1.2 & $97.87 \%$ & $97.32 \%$ & $0.55 \%$ & 1.2 & $94.78 \%$ & $94.18 \%$ & $0.59 \%$ \\
\hline 1.6 & $95.79 \%$ & $93.52 \%$ & $2.27 \%$ & 1.6 & $88.01 \%$ & $85.27 \%$ & $2.74 \%$ \\
\hline 2.0 & $94.42 \%$ & $91.50 \%$ & $2.92 \%$ & 2.0 & $81.95 \%$ & $77.79 \%$ & $4.16 \%$ \\
\hline 2.4 & $93.83 \%$ & $89.96 \%$ & $3.87 \%$ & 2.4 & $77.43 \%$ & $73.02 \%$ & $4.40 \%$ \\
\hline
\end{tabular}

We now analyze the performances of the exact method BBAlgorithm and compare its solutions with those of BendAlgorithm. The running time required by BBAlgorithm is often too long for digraphs with more than 100 edges. Therefore, we decided to run BBAlgorithm only on the digraphs having up to 100 vertices and density up to 1.6; also, we stopped the computation in any case after a time $t$ of 3 minutes. In total we have $65.5 \%$ of the instances in BimodalAcyclic (i.e., 131 instances) and $40.5 \%$ of the instances in Any (i.e., 81 instances). For the Rome digraphs, the percentage of instances solved within $t$ is $48.7 \%$ (i.e., 487 
instances). For all solved instances, we compared the size of the optimal solutions with those computed by BendAlgorithm to get an estimation of how much BendAlgorithm approximates the optimum: BendAlgorithm achieves the optimum on $92.37 \%$ of the BimodalAcyclic instances, on $67.9 \%$ of the Any instances, and on $83.98 \%$ of the Rome instances. In the remaining $7.63 \%$ of BimodalAcyclic instances, the optimum has in the average $2.35 \%$ edges more than the solution of BendAlgorithm; this percentage grows to $4.02 \%$ for the remaining $32.1 \%$ of the Any instances, and to $3.78 \%$ for the remaining $16.02 \%$ of the Rome instances. BendAlgorithm is therefore a good approximation of the optimum in many cases.

\section{References}

1. Bertolazzi, P., Di Battista, G., Didimo, W.: Quasi-upward planarity. Algorithmica 32(3), 474-506 (2002)

2. Bertolazzi, P., Di Battista, G., Liotta, G., Mannino, C.: Upward drawings of triconnected digraphs. Algorithmica 6(12), 476-497 (1994)

3. Bertolazzi, P., Di Battista, G., Mannino, C., Tamassia, R.: Optimal upward planarity testing of single-source digraphs. SIAM J. Comput. 27, 132-169 (1998)

4. Chan, H.: A parameterized algorithm for upward planarity testing. In: Albers, S., Radzik, T. (eds.) ESA 2004. LNCS, vol. 3221, pp. 157-168. Springer, Heidelberg (2004)

5. Di Battista, G., Eades, P., Tamassia, R., Tollis, I.G.: Graph Drawing. Prentice Hall, Upper Saddle River, NJ (1999)

6. Di Battista, G., Garg, A., Liotta, G., Tamassia, R., Tassinari, E., Vargiu, F.: An experimental comparison of four graph drawing algorithms. Computational Geometry: Theory and Applications 7, 303-326 (1997)

7. Didimo, W.: Upward planar drawings and switch-regularity heuristics. Journal of Graph Algorithms and Applications 10(2), 259-285 (2006)

8. Didimo, W., Giordano, F., Liotta, G.: Upward spirality and upward planarity testing. In: Healy, P., Nikolov, N.S. (eds.) GD 2005. LNCS, vol. 3843, pp. 117-128. Springer, Heidelberg (2006)

9. Garey, M.R., Johnson, D.S.: Comput. and Intract. Freeman and Co, San Francisco (1979)

10. Garg, A., Tamassia, R.: On the computational complexity of upward and rectilinear planarity testing. SIAM J. Comput. 31(2), 601-625 (2001)

11. Healy, P., Lynch, K.: Fixed-parameter tractable algorithms for testing upward planarity. International Journal of Foundations of Computer Science 17(5) (2006)

12. Hutton, M.D., Lubiw, A.: Upward planarity testing of single-source acyclic digraphs. SIAM J. Comput. 25(2), 291-311 (1996)

13. Lichtenstein, D.: Planar formulae and their uses. SIAM J. Comput. 11(2), 329-343 (1982)

14. Papakostas, A.: Upward planarity testing of outerplanar dags. In: Tamassia, R., Tollis, I(Y.) G. (eds.) GD 1994. LNCS, vol. 894, pp. 298-306. Springer, Heidelberg (1995) 